Aims/Objectives/Background We aim to examine senior managers' perspectives on funding mechanisms used to implement the policy and experiences of success or challenges in introducing models of using GPs in or alongside emergency departments. Health policy in England has advocated the use of primary care clinicians at emergency departments to address pressures from rising attendances. However, implementing large systemic changes such as placing GPs in or alongside emergency departments requires significant funding, consideration of the opportunity costs of the alternative uses of such funding, an available workforce and evidence of how it should be used. Our findings will inform policy adaptation and service development to improve the healthcare provided to patients by providing new evidence of the reported experiences of adopting models of using GPs in or alongside emergency departments.

Methods/Design The perspectives of senior clinical, business and finance managers with responsibility for emergency department services and on-site primary care service implementation were investigated in semi-structured interviews with 31 managers at 12 type- 1 emergency departments in England and Wales. Emergency departments operated one of three GP models or had prior experience of implementing a GP model. Interviews were thematically analysed.

Results/Conclusions Successful GPs models in emergency departments were perceived to be reliant on well-organised and unified funding mechanisms, appropriate staffing and governance, and consideration of population demands and needs. Funding mechanisms and the flow of funds were reported as complex, the most efficient mechanisms were described at departments where funding was unified, in collaboration with health and community care services. Staffing with local, experienced GPs was important. There were also cautions from experiences with private locum providers. Our findings contribute to debates about implementing policy on how primary care clinicians are effectively and safely deployed in emergency departments and how local context should be considered.

\section{WHAT GOES BUMP IN THE NIGHT? A LITERATURE REVIEW AND NOISE EXPOSURE EXPERIMENT IN A UK PAEDIATRIC EMERGENCY DEPARTMENT}

${ }^{1}$ Lucy Hall, ${ }^{2}$ Sophie Dando, ${ }^{2}$ Anthony Hanks. ${ }^{1}$ Student at Cardiff University; ${ }^{2}$ University Hospital UK

10.1136/emj-2020-rcemabstracts.32

Aims/Objectives/Background In the Emergency department (ED), noise is a frequent and often unavoidable consequence of work undertaken and levels can often be raised during the day and night. Raised ambient noise levels have potential implications for the workforce, patients and relatives.

Investigation into the problem of noise levels in the ED follows feedback from a young patient who couldn't sleep during a prolonged stay. His complaint focused on loud, irregular banging noises such as those from closing bins that kept him awake.

The team felt work should be done to see if it was a wider spread problem or just isolated to his case. A simple sound recording experiment and literature search was conducted.
Methods/Design The literature search was conducted using electronic/online databases (Medline; Cochrane library) with a fixed date range and specific inclusion criteria.

The noise exposure experiment was conducted using a verified phone app to record the sound levels. They were measured at 3 times, during a night shift, in the paediatric emergency department of UHW. All measurements were at a fixed distance and were averaged and compared with WHO recommendations.

Results/Conclusions There are many sources of noise pollution in the ED, some are unavoidable for safety and clinical reasons.

The literature review produced a small number of papers all of which found that sound levels were raised above recommended levels. Similarly, all the sounds measured in the ED also exceeded the recommendations.

The most consistent finding across the papers, matched by findings from recordings, was that human behavioural modification is an easy and effective way to reduce noise levels.

There are simple steps that can be taken to reduce and eliminate sounds

Raising awareness regarding this problem is of great importance and focussing future work on assessing the impact in younger patients within the Emergency Department is paramount.

\section{A CLASSIFICATION OF PRIMARY CARE PATHWAYS IN EMERGENCY DEPARTMENTS: A MULTI-METHODS STUDY COMPRISING CROSS-SECTIONAL SURVEY; SITE VISITS WITH OBSERVATIONS; SEMI-STRUCTURED AND INFORMAL INTERVIEWS}

${ }^{1}$ Michelle Edwards, ${ }^{1}$ Alison Cooper, ${ }^{1}$ Davies Freya, ${ }^{1}$ Andrew Carson-Stevens, ${ }^{2}$ Thomas Hughes, ${ }^{3}$ Niro Siriwardena, ${ }^{4}$ Helen Snooks, ${ }^{1}$ Adrian Edwards. ${ }^{1}$ Cardiff University; ${ }^{2}$ John Radcliffe Hospital; ${ }^{3}$ Lincoln University; ${ }^{4}$ Swansea University

\subsection{6/emj-2020-rcemabstracts.33}

Aims/Objectives/Background We aim to describe and classify the predominant streaming pathways on arrival in Emergency Departments (EDs) in England and Wales and explain how they operate in different models of emergency department primary care services. Recent policy has encouraged a method whereby nurses stream from the emergency department front door to GPs working in a separate GP service operating within or alongside an ED. However, there is variation in methods of assessing and streaming patients on arrival at EDs. Conflated terminology causes difficulties in assessing relative performance, improving quality or gathering evidence about safety, clinical effectiveness. Our findings present a new classification of current streaming pathways from emergency departments to primary care services.

Methods/Design We used a multi-stage method approach, including an online survey completed by 77 EDs across England \& Wales, interviews with 21 clinical leads, and finally, undertaking case studies of 13 EDs. Qualitative data were triangulated and analysed using a framework analysis approach.

Results/Conclusions The most common ED pathways to primary care services were: front door streaming before ED registration; streaming inside the $\mathrm{ED}$; or without streaming but GPs selecting patients. Pathways were often adapted, to 